\title{
The Middle Equitable Dominating Graphs
}

\author{
Anwar Alwardi ${ }^{1}$, Nandappa D. Soner ${ }^{1}$, Ahmad N. Al-Kenani ${ }^{2}$ \\ ${ }^{1}$ Department of Studies in Mathematics, University of Mysore, Mysore, India \\ ${ }^{2}$ Department of Mathematics, King Abdulaziz University, Jeddah, KSA \\ Email: aalkenani10@hotmail.com
}

Received April 20, 2012; revised May 25, 2012; accepted June 15, 2012

\begin{abstract}
Let $G=(V, E)$ be a graph and $A(G)$ is the collection of all minimal equitable dominating set of $G$. The middle equitable dominating graph of $G$ is the graph denoted by $M_{e d}(G)$ with vertex set the disjoint union of $V \cup A(G)$ and $(u, v)$ is an edge if and only if $u \cap v \neq \phi$ whenever $u, v \in A(G)$ or $u \in v$ whenever $u \in v$ and $v \in A(G)$. In this paper, characterizations are given for graphs whose middle equitable dominating graph is connected and $K_{p} \subseteq M_{e d}(G)$. Other properties of middle equitable dominating graphs are also obtained.
\end{abstract}

Keywords: Eqitable Domination Number; Middle Equitable Dominating Graph; Intersection Graphs

\section{Introduction}

All the graphs considered here are finite and undirected with no loops and multiple edges. As usual $p=|V|$ and $q=|E|$ denote the number of vertices and edges of a graph $G$, respectively. In general, we use $\langle X\rangle$ to denote the subgraph induced by the set of vertices $X$ and $N(v)$ and $N[v]$ denote the open and closed neighbourhoods of a vertex $\mathrm{v}$, respectively. A set $\mathrm{D}$ of vertices in a graph $\mathrm{G}$ is a dominating set if every vertex in $V-D$ is adjacent to some vertex in $D$. The domination number $\gamma(G)$ is the minimum cardinality of a dominating set of $G$. For terminology and notations not specifically defined here we refer reader to [1]. For more details about parameters of domination number, we refer to [2], and [3].

A subset $D$ of $V(G)$ is called an equitable dominating set of a graph $G$ if for every $u \in(V-D)$, there exists a vertex $v \in D$ such that $u v \in E(G)$ and $|\operatorname{deg}(u)-\operatorname{deg}(v)| \leq 1$. The minimum cardinality of such a dominating set is denoted by $\gamma_{e}(G)$ and is called equitable domination number of $G . D$ is minimal if for any vertex $u \in D, D-\{u\}$ is not a equitable dominating set of $G$. A subset $S$ of $V$ is called a equitable independent set, if for any $u \in S, v \notin N_{e}(u)$, for all $v \in S-\{u\}$. If a vertex $u \in V$ be such that

$|\operatorname{deg}(u)-\operatorname{deg}(v)| \geq 2$ for all $v \in N(u)$ then $u$ is in each equitable dominating set. Such vertices are called equitable isolates. The equitable neighbourhood of $u$ denoted by $N_{e}(u)$ is defined as

$N_{e}(u)=\{v \in V / v \in N(u),|\operatorname{deg}(u)-\operatorname{deg}(v)| \leq 1\}$ and

$u \in I_{e} \Leftrightarrow N_{e}(u)=\varnothing$. The cardinality of $N_{e}(u)$ is denoted by $\operatorname{deg}_{e}(u)$. The maximum and minimum equitable degree of a point in $G$ are denoted respectively by $\Delta_{e}(G)$ and $\delta_{e}(G)$. That is $\Delta_{e}(G)=\max a_{u \in V(G)}\left|N_{e}(u)\right|$, $\delta_{e}(G)=\min _{u \in V(G)}\left|N_{e}(u)\right|$. An edge $e=u v$ called equitable edge if $|\operatorname{deg}(u)-\operatorname{deg}(v)| \leq 1\}$, for more details about equitable domination number see [4]. Let $S$ be a finite set and let $F=\left\{S_{1}, S_{2}, \cdots, S_{n}\right\}$ be a partition of $S$. Then the intersection graph $\Omega(F)$ of $F$ is the graph whose vertices are the subsets in $F$ and in which two vertices $S_{i}$ and $S_{j}$ are adjacent if and only if $S_{i} \cap S_{j} \neq \phi, i \neq j$. Kulli and Janakiram introduced new classes of intersection graphs in the field of domination theory see [5-8].

The purpose of this paper is to introduce a new class of intersection graphs in the field of domination theory as follows:

Let $G=(V, E)$ be a graph and $S$ be the collection of minimal equitable dominating set of $G$. The middle equitable dominating graph of $G$ is the graph denoted by $M_{e d}(G)$ with vertex set the disjoint union $V(G) \cup S$ and $u v$ is an edge if and only if $u \cap v \neq \phi$ whenever $u, v \in S$ or $u \in v$ whenever $u \in V(G)$ and $v \in S$.

Example. Let $G$ be a graph as in Figure 1(a), then the equitable dominating sets are $\{1,4,5\},\{2,4,5\},\{3,4,5\}$ and the The middle equitable dominating graph shown in Figure 1(b).

\section{Main Results}

Theorem 2.1. A graph $G$ with $p \geq 3$ vertices is without equitable edge (equitable edge-free graph) if and only 


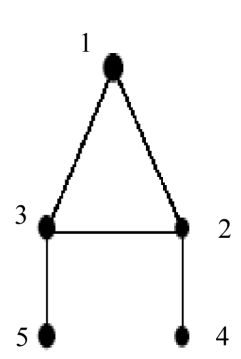

(a)

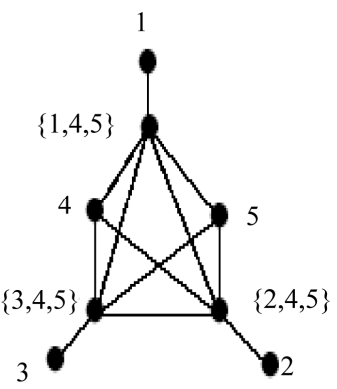

(b)
Figure 1. Example.

if $M_{e d}(G) \cong K_{1, p+1}$.

Proof. Suppose that $M_{e d}(G) \cong K_{1, p+1}$ and if its possible that $G$ has equitable edge $u v$. Then $G$ has at least two minimal equitable dominating set $(p \geq 3)$, a contradiction.

Conversely, assume that $G$ is equitable edge-free graph with $p$ vertices. Then clear all the vertices are equitable isolated vertices, that is there is only one minimal equitable dominating set contains all the vertices and according the definition of $M_{e d}(G)$, we get $M_{e d}(G) \cong K_{1, p+1}$.

Corollary 2.2. If $M_{e d}(G) \cong K_{1,2}$ if and only if $G \cong K_{2}$.

Corollary 2.3. If $M_{e d}(G) \cong K_{r, m}$, where $|r-m| \geq 2$, then $\gamma_{e}\left(M_{e d}(G)\right)=p+1$.

Corollary 2.4. Let $G$ be complete multi bipartite graph $K_{n_{1}, n_{2}, \ldots n_{t}}$, where $\left|n_{i}-n_{i} \geq 2\right|$, where $i, j=1,2, \cdots t$, then $M_{e d}(G) \cong K_{1}, \sum_{i=1}^{t} n_{i}$.

Proposition 2.5. $M_{e d}(G)=p K_{2}$ if and only if $G=K_{p}$.

Proof. Suppose that $G=K_{p}$. Then clearly each vertex in $G$ will form a minimal equitable dominating set. Hence $M_{e d}(G)=p K_{2}$.

Conversely, suppose $M_{e d}(G)=p K_{2}$ and $G \neq K_{p}$. Then there exists at least one minimal equitable dominating set $S$ containing two vertices of $G$. Then $S$ will form $p_{3}$ in $M_{e d}(G)$.

Proposition 2.6. If $G=K_{p}$, then $\gamma\left(M_{e d}(G)\right)=\gamma_{e}\left(M_{e d}(G)\right)=p$.

Proof. The proof coming directly from Proposition 2.5 .

Theorem 2.7. Let $G$ be a graph with $p$ vertices and $q$ edges. $M_{e d}(G)$ is a graph with $2 P$ vertices and $p$ edges if and only if $G \cong K_{p}$.

Proof. If $G \cong K_{p}$, then that is clear the $M_{e d}(G)$ is a graph with $2 P$ vertices and $p$ edges.

Conversely, let $M_{e d}(G)$ be $(2 p, p)$-graph. Since only the graph $p K_{2}$ is of $2 p$ vertices and $p$ edges, then by Proposition $2.5 G \cong K_{p}$.

Theorem 2.8. Let $G$ be a graph with $p$ vertices and $q$ edges. $M_{e d}(G)$ is a graph with $P+1$ vertices and $p$ edges if and only if $G$ is equitable edge-free graph.

Proof. Let $G$ be equitable edge-free graph. Then by Theorem $2.1 M_{e d}(G)$ is a graph with $P+1$ vertices and $p$ edges.

Conversely, let $M_{e d}(G)$ be a graph with $P+1$ vertices and $p$ edges that means $M_{e d}(G) \cong K_{1, p}$, and by Theorem $2.1 G$ is equitable edge-free graph.

Theorem 2.9. [1] Let $G$ be a graph, if $D$ is maximal equitable independent set of $G$, then $D$ also minimal equitable set.

Theorem 2.10. For any graph $G$ with at least two vertices $M_{e d}(G)$ is connected if and only if $\Delta_{e}(G)<p-1$.

Proof. Let $\Delta_{e}(G)<p-1$ and $u, v$ be any two vertices if there is minimal equitable dominating set $D$ containing $u$ and $v$, the $u, v$ are connected by the path $u D v$, and if there is no minimal equitable dominating containing both $u$ and $v$. Then there exists a vertex $w$ in $V(G)$ such that $w$ is neither adjacent to $u$ nor $v$. Let $D$ and $D$, be two maximal equitable independent set containing $u, w$ and $v, w$ respectively since every maximal equitable independent set is minimal equitable dominating set by Theorem 2.9, $u$ and $v$ are connected by the path $u D D^{\prime} v$. Thus $M_{e d}(G)$ is connected. Hence $M_{e d}(G)$ is connected.

Conversely, suppose $M_{e d}(G)$ is connected. let $\Delta_{e}(G)=p-1$ and let $u$ be a vertex such that $d_{e}(u)=$ $p-1$. Then $\{u\}$ is minimal equitable dominating set and $G$ has at least two vertices, it is clear that $G$ has no equitable isolated vertices, then $V-D$ containing minimal equitable dominating set say $D^{\prime}$. Since

$D \cap D^{\prime}=\phi$ in $M_{e d}(G)$ there is no path joining $u$ to any vertex of $V-D$, this implies that $M_{e d}(G)$ is disconnected, a contradiction. Hence $\Delta_{e}(G)<p-1$.

Corollary 2.11. Let $G=(V, E)$ be a graph and $u, v$ any two vertices in $V(G)$. Then $d(u, v)_{M_{e d}(G)} \leq 3$, where $d(u, v)_{M_{e d}(G)}$ is the distance between the vertex $u$ and $v$ in the graph $M_{e d}(G)$.

Theorem 2.12. For any graph $G, K_{t} \subseteq M_{e d}(G)$ if and only if $G$ contains one equitable isolated vertex, where $t$ is the number of minimal equitable dominating set in $G$.

Proof. If $G$ has equitable isolated vertex then the subgraph of $M_{e d}(G)$ which induced by the set of all minimal equitable dominating sets of $G$ is complete graph $K_{t}$. Hence $K_{p} \subseteq M_{e d}(G)$.

Conversely, Suppose $K_{p} \subseteq M_{e d}(G)$, then it is clear that the vertices of $K_{t}$ are the minimal equitable dominating sets of $G$. Therefor there exists at least one equitable isolated vertex in $G$.

Theorem 2.13. For any graph $G, M_{e d}(G)$ is either connected or it has at least one component which is $K_{2}$. 
Proof. If $\Delta_{c n}(G)<p-1$, then by Theorem $2.10 M_{e d}$ is connected, and By Proposition 2.5, if $G$ is complete $M_{e d}=p K_{2}$ which is connected. Hence we must prove the case $\delta_{e}(G)<\Delta_{e}(G)=p-1$.

Let $\left\{u_{1} \cdots u_{s}\right\}$ be the set of vertices in $G$ such that $d_{e}\left(u_{i}\right)=p-1$, where $i=1, \cdots, t$, then it is clear $\left\{u_{i}\right\}$ is minimal equitable dominating set. Then each vertex $u_{i}$ with the minimal equitable dominating set $\left\{u_{i}\right\}$ $i=1, \cdots s$ form component isomorphic to $K_{2}$. Hence it has at least one component which is $K_{2}$.

Theorem 2.14. For any graph $G$ with $\Delta_{e}(G)<p-1$, $\operatorname{diam}\left(M_{e d}(G)\right) \leq 5$.

Proof. Let $G$ be any graph and $\Delta_{e}(G)<p-1$. Then by Theorem 2.10, $M_{e d}(G)$ is connected, suppose $u, v$ be any two vertices in $M_{e d}(G)$, then we have the following cases:

Case 1: Let $u, v$ be any two vertices in $V(G)$. Then by corollary 2.11, $d(u, v)_{M_{e d}(G)} \leq 3$.

Case 2: Suppose $u \in V(G)$ and $v=D$ be a minimal equitable dominating set in $G$, If $u \in D$ then $d(u, v)=1$ and if $u \notin D$ then there exists a vertex $w \in D$ adjacent to $u$. Hence

$d(u, v)_{M_{e d}(G)}=d(u, w)_{M_{e d}(G)}+d(w, v)_{M_{e d}(G)}$ and from corollary 2.11 we have $d(u, w)_{M_{e d}(G)} \leq 3$. Hence

$d(u, v)_{M_{e d}(G)} \leq 4$.

Case 3: Suppose that both $u$ and $v$ are not in $V(G)$, and $u=D, v=D^{\prime}$ are two minimal equitable dominating sets if $D$ and $D^{\prime}$ are adjacent, then $d(u, v)_{M_{e d}(G)}=1$, suppose that $D$ and $D^{\prime}$ are not adjacent then every vertex $w \in D$ is adjacent to some vertex $x \in D^{\prime}$ and vice versa. Hence

$d(u, v)=d(u, w)+d(w, x)+d(x, v) \leq 5$ Hence $\operatorname{diam}\left(M_{e d}(G)\right) \leq 5$.

Theorem 2.15. Let $G$ be a graph. Then $d_{e}\left(M_{e d}(G)\right)=2$ if and only if $G=K_{p}$ or $G=\overline{K_{2}}$, where $d_{e}\left(M_{e d}(G)\right)$ is the equitable domatic number of the graph $M_{e d}(G)$.

Proof. If $G=K_{p}$ or $G=\overline{K_{2}}$, then $M_{e d}(G)=p K_{2}$ or $G=K_{1,2}$ by Proposition 2.5. Hence $d_{c n}\left(M_{e d}(G)\right)=2$. The converse is obvious.

Theorem 2.16. For any graph $G$ with at least one equitable isolated vertex,

\section{$\beta_{e}\left(M_{e d}(G)\right)=\beta\left(M_{e d}(G)\right)=p$.}

Proof. Let $G$ be a graph of $p$ vertices, and has at least one equitable isolated vertex $w$. Then from the definition of $M_{e d}(G)$ if $u$ and $v$ any vertices in $G$ then $u$ and $v$ can not be adjacent in $M_{e d}(G)$, that is there is equitable independent set containing $w$ and of size $p$, and this equitable independent will be the maximum equitable independent because $w$ is adjacent for all the equitable independent sets. Therefore $\beta_{e}(G)=\beta(G)=p$.

Corollary 2.17. For any graph $G$ with at least one equitable isolated vertex, $\alpha_{e}\left(M_{e d}(G)\right)=\alpha\left(M_{e d}(G)\right)=|S|$, where $|S|$ is the number of minimal equitable dominating set in $G$

Proof. We have for any graph $G$ with $p$ vertices $\alpha_{c n}(G)+\beta_{c n}(G)=\alpha(G)+\beta(G)=p$, and from [1]. Theorem 3.8.4 corollary is proved.

Theorem 2.18. For any graph $G$, $d_{e}(G)=\beta_{e}\left(M_{e d}(G)\right)$, if and only if $G$ is complete graph.

Proof. Let $G$ be complete graph $K_{p}$. Then from Theorem $2.16 \beta_{e}\left(M_{e d}(G)\right)=p$, and we have $d_{e}(G)=p$. Hence $d_{e}(G)=\beta_{e}\left(M_{e d}(G)\right)$.

Conversely, suppose $d_{e}(G)=\beta_{e}\left(M_{e d}(G)\right)$. From Theorem $2.16 \beta_{e}\left(M_{e d}(G)\right)=p$ implies that $d_{e}(G)=p$. Hence $G=K_{p}$.

\section{Acknowledgements}

The authors wish to thank the referees for their helpful comments.

\section{REFERENCES}

[1] K. D. Dharmalingam, "Studies in Graph Theorey-Equitable Domination and Bottleneck Domination,” Ph.D. Thesis, Madurai Kamaraj University, Madurai, 2006.

[2] F. Harary, “Graph Theory,” Addison-Wesley, Boston, 1969.

[3] T. W. Haynes, S. T. Hedetniemi and P. J. Slater, "Fundamentals of Domination in Graphs,” Marcel Dekker, Inc., New York, 1998.

[4] V. R. Kulli and B. Janakiram, "The Minimal Dominating Graph," Graph Theory Notes of New York, Vol. 28, Academy of Sciences, New York, 1995, pp. 12-15.

[5] V. R. Kulli, B. Janakiram and K. M. Niranjan, "The Common Minimal Dominating Graph,” Indian Journal of Pure and Applied Mathematics, Vol. 27, No. 2, 1996, pp. 193196.

[6] V. R. Kulli, B. Janakiram and K. M. Niranjan, "The Vertex Minimal Dominating Graph,” Acta Ciencia Indica, Vol. 28, 2002, pp. 435-440.

[7] V. R. Kulli, B. Janakiram and K. M. Niranjan, "The Dominating Graph,” Graph Theory Notes of New York, Vol. 46, 2004, pp. 5-8.

[8] H. B. Walikar, B. D. Acharya and E. Sampathkumar, "Recent Developments in the Theory of Domination in Graphs," MRI Lecture Notes in Mathematices, Vol. 1, Mehta Research Institute, Alahabad, 1979. 